\title{
Vestibular Disorder, CTCAE
}

National Cancer Institute

\section{Source}

National Cancer Institute. Vestibular Disorder, CT CAE. NCI Thesaurus. Code C143936.

A disorder characterized by dizziness, imbalance, nausea, and vision problems. 